\title{
LA HUELLA HÍDRICA COMO UNA ESTRATEGIA DE EDUCACIÓN AMBIENTAL ENFOCADA A LA GESTIÓN DEL RECURSO HÍDRICO: EJERCICIO CON COMUNIDADES RURALES DE VILLAVICENCIO ${ }^{1}$
}

\author{
SANDRA MILENA DELGADO-GARCÍA ${ }^{2-3}$, JUAN MANUEL \\ TRUJILLO-GONZÁLEZ ${ }^{2-4}$, MARCO AURELIO TORRES- \\ MORA $^{2-5}$
}

Recibido el 7 de diciembre de 2012 y aprobado el 21 de febrero de 2013

\section{RESUMEN}

De acuerdo con Tréllez (2002), la educación ambiental comunitaria es un campo abierto al pensamiento y a la acción constructiva, en donde los resultados pueden convertirse gradualmente en propuestas creativas para un futuro diferente; de esta forma, la educación ambiental permite flexibilizar el pensamiento, crear escenarios y construir procesos orientadores para el cambio. Este estudio tiene el propósito de generar cambios en la percepción con respecto al consumo de agua indirecta en los hogares, por medio de la educación ambiental y a través de la metodología investigación-acción, con talleres participativos en donde se calculó la huella hídrica indirecta por consumo de alimentos. A partir de este cálculo, las comunidades mostraron un cambio sobre el consumo de agua, y de ahí generaron propuestas para mejorar su relación con el recurso. Esta estrategia de educación ambiental implementada a través de la huella hídrica, permitió generar el inicio de la apropiación por parte de las comunidades con respecto a la gestión del recurso hídrico, y generar en los líderes la responsabilidad de transmitir lo aprendido a los pobladores de su vereda.

\section{PALABRAS CLAVE}

Educación ambiental, huella hídrica,comunidad, percepción, investigación-acción.

THE WATER FOOTPRINT AS AN ENVIRONMENTAL EDUCATION STRATEGY FOCUSED ON THE MANAGEMENT OF WATER RESOURCES: STUDY CONDUCTED WITH RURAL COMMUNITIES FROM VILLAVICENCIO

\begin{abstract}
According to Tréllez (2002), community-based environmental education is an open field to thought and to constructive action whose individual results can gradually become creative proposals for a different future; in this way, environmental education allows for the flexibility of thoughts, the creation of scenarios, and the development of processes oriented towards change. The purpose of this study is to produce a change in relation to the indirect consumption of water in homes, through
\end{abstract}


environmental education, by means of Action research with participative workshops in which the indirect water footprint for food consumption was calculated. After this calculation, communities showed a noticeable change regarding their consumption of water, developing proposals to improve their relationship with this resource. This environmental education strategy, implemented through the water footprint, has allowed for the communities to begin to appropriate management of water resources and to develop responsibility in the leaders to communicate what has been learned to other members within their respective communities.

\section{KEY WORDS}

Environmental education, water footprint, community, perception, action-research.

\section{INTRODUCCIÓN}

En la actualidad existen desequilibrios entre la oferta y la demanda del recurso agua y/o entre sus diversos usos generados por el aumento de la población, lo cual prende las alarmas de instituciones internacionales preocupadas por la gestión del agua, dado que gran parte de la población no tiene conciencia del uso que se le está dando al recurso. Esto hace necesario mejorar nuestro conocimiento acerca de la gestión y cuidado del recurso hídrico (Vélez-Upegui \& Correa-Velásquez, 2002); es por esta razón que la educación ambiental, según Alegre (2010), debe estar orientada a la comunidad, involucrando a los individuos en un proceso activo para resolver los problemas en el contexto de sus realidades específicas, fomentando la participación y la iniciativa para superar las dificultades que los afectan, pero esencialmente para evitar crear nuevas situaciones de conflicto.

En las propuestas de educación ambiental es necesaria la implementación de estrategias para que las comunidades se apropien de la importancia y tomen conciencia del uso que están dando a los recursos naturales; en este caso, al agua que se consume en los hogares. A partir del concepto de huella hídrica $(\mathrm{HH})$ propuesto por Hoekstra y Hung en 2002, que permite estimar el agua que ha sido necesaria en la producción de bienes y servicios, se plantearon talleres participativos con comunidades rurales del municipio de Villavicencio, con las que se pretendió generar un cambio de percepción en el uso del agua a través del cálculo de la HH por consumo de alimentos, propiciando espacios de autorreflexión en donde se genere un cambio en las relaciones hombre, naturaleza y sociedad.

Tréllez (2006), plantea que las comunidades necesitan llevar a cabo procesos de autogestión ambiental comunitaria para el desarrollo sustentable, que amplíen su visión y les permitan orientar una acción permanente de análisis y planificación respecto a la situación ambiental de su población. La 
autogestión se enfoca en la búsqueda y realización de procesos gestados en el seno de las mismas comunidades, sobre la base de sus experiencias y con la mira puesta en la construcción del futuro que consideran deseable.

Este tipo de procesos enmarcados dentro de la educación popular ambiental, propician que los propios sujetos puedan realizar una lectura crítica de la realidad en que se encuentran, lo cual les posibilita identificar problemáticas, proponer alternativas de acción y construir o reconstruir propuestas factibles de solución. De esta manera, no se pretende la competitividad, sino la solidaridad fundada en el reconocimiento de las diferencias y en la igualdad de oportunidades de realización humana (Calixto, 2010).

\section{MATERIALES Y MÉTODOS}

El desarrollo de este trabajo se llevó a cabo bajo la metodología de la investigación-acción de Elliott (1993), cuyo objetivo esencial es el de operar un cambio en un medio (en la gente y en el medio ambiente) y cuya dinámica es participativa, implicando los diferentes actores de una situación por transformar (Sauvé, 2004).

Se contó con la participación de 35 líderes comunitarios de los corregimientos 4 y 7 de Villavicencio, con los cuales se implementó la estrategia educativa a través de talleres, con el objeto de analizar los cambios en la percepción que tienen sobre el uso del recurso hídrico.

El primer taller, indagó sobre ¿Cuánta agua consume normalmente en un día?, seguidamente se expuso el concepto de huella hídrica, se calculó la HH individual y comunitaria, para lo cual se entregó un listado de productos alimenticios con su respectiva HH (Tabla1). Con base en lo anterior, en el segundo taller, nuevamente se indagó sobre los resultados obtenidos y la nueva percepción de uso del recurso agua (Figura 1).

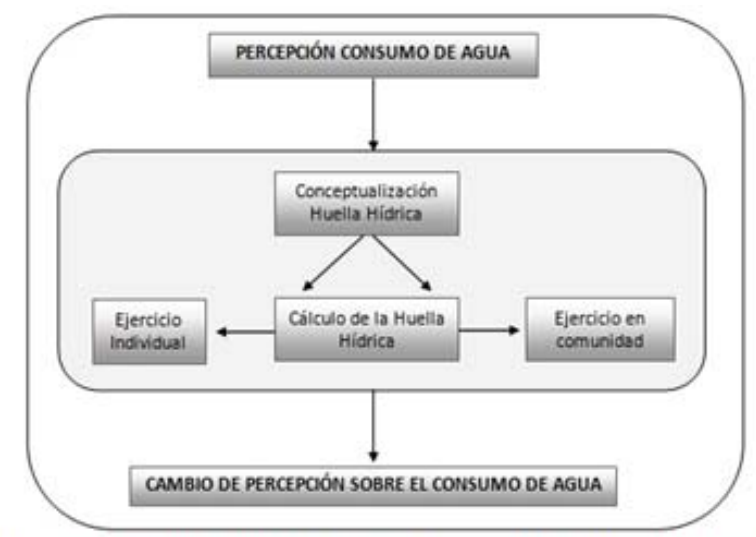

Figura 1. Esquema de la estrategia de educación ambiental implementada. 
Tabla 1. Huella Hídrica de algunos productos alimenticios

\begin{tabular}{|c|c|c|}
\hline PRODUCTO & $\begin{array}{c}\text { LITROS DE } \\
\text { AGUA }\end{array}$ & FUENTE \\
\hline Taza de té & 30 & Chapagain \& Hoekstra (2003) \\
\hline $100 \mathrm{~g}$ de chocolate & 2400 & Hoekstra (2008) \\
\hline Un tomate & 13 & Hoekstra \& Chapagain (2007) \\
\hline Taza de café & 140 & Chapagain \& Hoekstra (2003) \\
\hline Una lb de azúcar & 750 & Hoekstra (2008) \\
\hline Una cerveza & 105 & Hoekstra \& Chapagain (2007) \\
\hline Una naranja $(100 \mathrm{~g})$ & 50 & Hoekstra \& Chapagain (2007) \\
\hline Una manzana $(100 \mathrm{~g})$ & 70 & Hoekstra \& Chapagain (2007) \\
\hline Un paquete de papas & 185 & Hoekstra \& Chapagain (2007) \\
\hline Un huevo & 135 & Hoekstra \& Chapagain (2007) \\
\hline Un banano $(100 \mathrm{~g})$ & 80 & Hoekstra (2008) \\
\hline Una papa $(100 \mathrm{~g})$ & 25 & Hoekstra \& Chapagain (2007) \\
\hline Una tajada de pan & 40 & Hoekstra \& Chapagain (2007) \\
\hline Una hamburguesa & 2400 & Hoekstra \& Chapagain (2007) \\
\hline Un vaso de leche & 200 & Hoekstra \& Chapagain (2007) \\
\hline Una copa de vino & 120 & Hoekstra \& Chapagain (2007) \\
\hline Un vaso de jugo de naranja & 190 & Hoekstra \& Chapagain (2007) \\
\hline Una tajada de queso & 50 & Hoekstra \& Chapagain (2007) \\
\hline Un kg de pasta & 1924 & Aldaya \& Hoekstra (2010) \\
\hline Una pizza & 1216 & Aldaya \& Hoekstra (2010) \\
\hline Un kg de carne de res & 15500 & Hoekstra (2008) \\
\hline Un kg de cerdo & 4800 & Hoekstra (2008) \\
\hline Un kg de arroz & 1193 & Trujillo (2012) \\
\hline Un kg de mango & 1600 & Hoekstra (2008) \\
\hline Un kg de maíz & 900 & Hoekstra (2008) \\
\hline $100 \mathrm{~g}$ de pollo & 3900 & Hoekstra (2008) \\
\hline Un kg de calabaza & 240 & Hoekstra (2008) \\
\hline Un kg de lechuga & 130 & Hoekstra (2008) \\
\hline Un kg de repollo & 200 & Hoekstra (2008) \\
\hline
\end{tabular}

Finalmente se realizó un conversatorio donde se expusieron las experiencias logradas con las actividades desarrolladas.

\section{RESULTADOS Y DISCUSIÓN}

En la Tabla 2, se presentan algunas de las precepciones antes y después de la implementación de la estrategia de educación ambiental, donde se observa un cambio en la percepción sobre el uso del agua en sus hogares, donde reconocen cuánta agua ha sido empleada únicamente en la producción de alimentos sin considerar otros bienes y servicios de consumo. 
Tabla 2. Percepciones de la comunidad *

\begin{tabular}{|c|c|}
\hline PERCEPCION ANTES & PERCEPCION DESPUES \\
\hline $\begin{array}{l}\text { "Mi consumo es alto ya q' utilizó } \\
\text { demasiada lavando la losa la ropa } \\
\text { bañándome lavando la casa avecesesajero } \\
\text { en el gasto osea la mal gasto". } \\
\text { Marcela Parrado García (Vereda la Vigía) }\end{array}$ & $\begin{array}{l}\text { "Realmente estoy muy asombrada al saber } \\
q \text { en un solo dia puedo llegar a consumir } \\
\text { toda esta cantidad de agua ya q gasto } \\
\text { mucho mas de lo que algún dia llegue a } \\
\text { imaginar. } \\
\text { Una propuesta seria dejar de consumir tan } \\
\text { seguido productos } q \text { impliquen un } \\
\text { exagerado gasto o consumo de agua". } \\
\text { Marcela Parrado Garcia (Vereda la Vigia) }\end{array}$ \\
\hline $\begin{array}{l}\text { "Me baño } 2 \text { veces al dia, al cepillarme los } \\
\text { dientes cierro la llave del } \mathrm{H}_{2} \mathrm{O} \text {, al lavarme } \\
\text { las manos cierro las llaves al enjabonarme } \\
\text { las manos. } \\
\text { Lavo en la lavadora los Domingos la ropa } \\
\text { de la semana realizo aseo en la casa los } \\
\text { Domingos por lo que durante la semana no } \\
\text { permanece nadie en el dia en la casa. } \\
\text { Promedio } \mathrm{H}_{2} \mathrm{O} \text { entre mi esposa y yo es de } \\
300 \text { Litros de } \mathrm{H}_{2} \mathrm{O} \text { semanal" } \\
\text { Jonnathan Santanilla (Vereda Santa } \\
\text { Helena) }\end{array}$ & $\begin{array}{l}\text { "Empezar a poner en practica en mi vida } \\
\text { una dieta valanceada y no comer cosas } \\
\text { innecesarias para empezar a aportar } \\
\text { buenas practicas a las personas que me } \\
\text { rodean tanto en mi vereda como en mi } \\
\text { familia." } \\
\text { Jonnathan Santanilla Perez } \\
\text { (Vereda Santa Helena) }\end{array}$ \\
\hline $\begin{array}{l}\text { "En mi casa el consumo de agua es } \\
\text { solamente el necesario para las labores } \\
\text { domésticas y de aseo. Todos nos turnamos } \\
x \text { dias para hervir el aguaque vamos a } \\
\text { consumir y nos vañamos una vez en el dia. } \\
\text { Siempre en las mañanas. Creo que } \\
\text { hacemos buen uso del agua ps nos } \\
\text { preocupamos } x \text { ahorrar el agua". } \\
\text { Sandy Hernandez (Vereda Alto de } \\
\text { Pompeya) }\end{array}$ & $\begin{array}{l}\text { "fue impresionante saber que solo } \\
\text { consumimos agua para nuestro sustento } \\
\text { diario directo sino que todo lo que } \\
\text { necesitamos para vivir, comer, y hasta para } \\
\text { vestimos en su proceso de elaboración } \\
\text { consumen agua de igual manera que } \\
\text { nosotros. Lo que quiere decir que no solo } \\
\text { consumimos bañándonos o lavarnos las } \\
\text { manos. Sino que también de forma } \\
\text { indirecta lo estamos haciendo en todo lo } \\
\text { que necesitamos" } \\
\text { Sandy Hernandez (Vereda Alto de } \\
\text { Pompeya) }\end{array}$ \\
\hline $\begin{array}{l}\text { "Me considero un consumidor medio de } \\
\text { agua, porque no siempre pero cuando hace } \\
\text { mucho calor me baño dos veces, tomo } \\
\text { bastante agua, tratamos de hacer solo } 2 \\
\text { lavadas a la semana y restringimos } \\
\text { bastante para no gastar agua, cuando se } \\
\text { lavaloza y patios" } \\
\text { Gabriel Andres Guevara (Vereda Santa } \\
\text { Rosa) }\end{array}$ & $\begin{array}{l}\text { "Ya sabiendo mas claramente cuales son } \\
\text { nuestros volúmenes de agua diarios es } \\
\text { bastante alarmante, buscare no consumir } \\
\text { habitualmente unos productos que no son } \\
\text { tan necesarios para mi alimentación, pero } \\
\text { que si demanden mucho consumo de agua } \\
\text { para su preparación" } \\
\text { Gabriel Andres Guevara (Vereda Santa } \\
\text { Rosa) }\end{array}$ \\
\hline $\begin{array}{l}\text { "Yo consumo bastante agua en el lavado } \\
\text { de ropa y lavando los veyculos de } \\
\text { transporte al diaconsumo mas de } 20 \text { litros } \\
\text { de agua". } \\
\text { Mauricio Moreno (Vereda Puerto Colombia) }\end{array}$ & $\begin{array}{l}\text { "Yo consumo mucha agua debo redusir el } \\
\text { consumo de agua por que } 31685 \text { es una } \\
\text { cantidad muy alta de agua apartir de oy me } \\
\text { comprometo a reducir". } \\
\text { Mauricio Moreno (Vereda Puerto Colombia) }\end{array}$ \\
\hline $\begin{array}{l}\text { "Consumo poco agua en casa se recicla el } \\
\text { agua de la lavadora esta es para el baño, } \\
\text { nos bañamos una vez al dia y tenemos una } \\
\text { tina donde cae el agua sucia que también } \\
\text { se utiliza en el baño, las matas y el piso, } \\
\text { por el alto consumo de energía al prender } \\
\text { la motobomba no consumimos mucha agua } \\
\text { adema, lavamos dia x medio creo que le } \\
\text { hacemos en casa un uso adecuado" } \\
\text { JoanaSanchez (Vereda Barcelona) }\end{array}$ & $\begin{array}{l}\text { "Por ignorancia peca el pueblo y eso me } \\
\text { paso a mi, controlare mas lo que consumo } \\
\text {, lo que compro, lo que visto, etc. Mas del } \\
98 \% \text { de este corregimiento o población no } \\
\text { sabemos de esto la huella hidrica creo que } \\
\text { hablare de esto no tan cientifico pero si lo } \\
\text { divulgare mas para comenzar a crear esta } \\
\text { conexión ambiental, hoy hidrate mi mente } \\
\text { en mas conocimiento lo que creia que } \\
\text { sabia es mas profundo de lo que pensé, } \\
\text { cuidez mas mi recurso hidrico". } \\
\text { Joana Sanchez (Vereda Barcelona) }\end{array}$ \\
\hline
\end{tabular}

A partir de la experiencia en el cálculo de la $\mathrm{HH}$, estos líderes ambientales han comenzado a generar propuestas, que a nivel local contribuyen en la gestión del recurso hídrico; tal como lo argumenta Tréllez (2006), la difusión de las experiencias permite que las comunidades resalten la importancia de proteger el patrimonio natural y se constituyan en un punto de partida para la creación de alternativas que definan nuevas orientaciones del desarrollo hacia mejores condiciones devida de la población. 
Dentro de las propuestas que la comunidad manifiesta, algunos líderes resaltan que se debe mantener una dieta balanceada, se debenconsumir alimentos que no demanden grandes cantidades de agua durante su producción, disminuir el consumo de carne roja y de cerveza, de igual forma tomar un solo baño al día, como también otras personas opinan que la mejor propuesta para contribuir en la gestión del recurso hídrico es difundiendo este tipo de información a las demás comunidades.

Los cambios en la percepción del uso del recurso hídricopor parte de los líderes, muestra lo complejo de su realidad y sus problemáticas, pero a la vez las múltiples posibilidades para proponer acciones tendientes a su mitigación. Este tipo de planteamiento es presentado por Alegre (2005), quien afirma que se debe capacitar a quienes están en puestos de gestión desde los ámbitos de gobierno y también al resto de los actores sociales locales, para lograr espacios de intercambio y de aportes, que se sustentenen un diálogo de saberes entre las diferentes miradas de los diversos actores comunitarios.

La nueva percepción por parte de los líderes comunitarios y su necesidad de irradiar a las comunidades el uso racional del recurso agua, es un resultado coincidente con algunas de las propuestas deLa Carta de Belgrado (1975), que señalan entre otras, a la educación como elemento de importancia en el proceso de cambio, a la enseñanza del conocimiento teórico y práctico,como también de valores y actitudes, como requerimiento para el mejoramiento ambiental (Leal, 2010).

\section{CONCLUSIONES}

La inclusión del concepto de huella hídrica y su cálculo como estrategia de educación ambiental por parte de los líderes de los corregimientos 4 y 7 del municipio de Villavicencio, permitió que estos líderes se apropiaran de la importancia del uso adecuado del recurso agua y de la responsabilidad adquirida para comunicar, replicar y transmitir las experiencias alcanzadas, como herramienta de gestión ambiental en su localidad.

Procesos de enseñanza-aprendizaje, como el de investigaciónacción en temas concretos como la huella hídrica, permiten un cambio en la manera como las comunidades perciben el uso de los recursosy también el cambio de actitudes para generar una mejor relación hombre, naturaleza y sociedad. Es importante concluir que esta estrategia de educación ambiental se acerca a las metas y objetivos planteados en Belgrado, con respecto a la necesidad de crear y desarrollar en el ser humano la conciencia, los conocimientos, las actitudes, las aptitudes, la participación y la necesidad de trabajar en forma individual y colectiva en la resolución de los problemas ambientales existentes. 


\section{AGRADECIMIENTOS}

Los autores agradecen a las comunidades de los corregimientos 4 y 7 del municipio de Villavicencio-Meta que participaron en esta iniciativa, y al profesor Miguel Ángel Venegas Rojas de la Universidad de los Llanos por su orientación.

\section{BIBLIOGRAFÍA}

- Aldaya, M. M. y Hoekstra, A. Y. (2010). El agua necesaria para los italianos comer pasta y pizza. Sistemas Agrícolas, 103, 351-360.

- Alegre, S. I. (2005). La Educación Ambiental como proceso. Revista Ambiente de Chicos, 3, 28-29. Secretaría de Ambiente y Desarrollo Sustentable de la Nación, UNESCO, PNUMA, UNICEF. República Argentina.

- _ (2010). La importancia de la participación ciudadana a través de la educación ambiental para la mitigación del cambio climático a nivel local. Desarrollo Local Sostenible, 3(7). Disponible en:http://www.eumed.net/rev/delos/07/sia.pdf [consultado el 20 de noviembre de 2012].

- Calixto Flores, R. (2010). Educación popular ambiental. Trayectorias [en línea], 12(30), 24-39 [citado 2012-1125]. Disponible en: http://redalyc.uaemex.mx/src/inicio/ArtPdfRed.jsp?i Cve $=60713488003$, ISSN 1405-8928.

- Chapagain, A. K. y Hoekstra, A. Y. (2003). El agua que se necesita para que el beber té holandés. Valor de Investigación del Agua, Serie de informe No. 15, UNESCO-IHE.

- Elliott, J. (1993). El cambio educativo desde la investigación-acción. Madrid: Morata.

- Hoekstra, A. Y. (2008). El agua neutral: la reducción y compensación de los impactos de huellas del agua. Valor del informe de investigación del agua, Serie No. 28, UNESCO-IHE.

- Hoeskstra, A. Y. y Chapagain, A. K. (2007). Huellas hídricas de las naciones: el uso del agua por la gente en función de su patrón de consumo. Gestión de recursos hídricos, 21(1), 35-48.

- Hoekstra, A. Y. \& Hung, P. Q. (2002). Virtual water trade: A quantification of virtual water flows between nations in relation to international crop trade'. Value of waterresearchreport, Serie No.11, UNESCO-IHE.

- La Carta de Belgrado. (1975). http://www.ambiente.gov.ar/infotecaea/descargas/b elgrado01.pdf.

- Leal Figueroa, P. (2010). Educación ambiental en chile: una necesidad ineludible. Una revisión bibliográfica. Educación y Humanidades, 1(1), 7-26. 
- Sauvé, L. (2004): «Uma cartografia das correntes em educação ambiental», en M. Sato y C. I. MouraCarvalho (eds.): Educação ambiental. Pesquisa, São Paulo, ARTMED, pp. 17-44.

- Tréllez Solis, E. (2002). La educación ambiental comunitaria y la retrospectiva: una alianza de futuro. Tópicos en educación ambiental, 4(10), 7-21.

- _ (2006). La formación ambiental comunitaria: una propuesta participativa. Disponible en: http://www.ambiente.gov.arlinfotecaea/descargas/tr ellez04.pdf[consultado 1 de diciembre de 2012].

- Trujillo, J. (2012). Huella hídrica de la producción de arroz paddy en los municipios del Meta. En: Memorias Taller "Experiencias y Expectativas en adaptación y mitigación del cambio climático en la región de la Orinoquía". NORECO - Nodo Regional de Cambio Climático Orinoquía.

- Vélez-Upegui, J. yCorrea-Velásquez, P. (2002). Implementación de un Sistema de Información Geográfica (SIG) para Apoyar la Gestión del Recurso Hídrico en la Cuenca de la Quebrada Chachafruto. XV Seminario Nacional de Hidráulica e Hidrología. Medellín.

1. Convenio de cooperación No. 5210955 Universidad de los Llanos - Ecopetrol S.A.: Desarrollo de procesos de formación y capacitación para la gestión ambiental en los municipios de influencia y operaciones de Ecopetrol S.A. (Villavicencio, Acacías y Castilla la Nueva) en el departamento del Meta, así como la construcción de tejido social a través de la recuperación histórica.

2. Grupo de Estudio Sistemas Productivos y Ambiente GESPA-, vinculado al Grupo de Investigación en Gestión Ambiental Sostenible -GIGAS-.Facultad de Ciencias Básicas e Ingeniería, Universidad de los Llanos, km 12 vía Puerto López, A.A. 110, Villavicencio, Meta, Colombia.

3. Licenciada en Producción Agropecuaria, Universidad de los Llanos. CM.Sc. en Gestión Ambiental Sostenible. sandritadelgado1821@gmail.com

4. Ingeniero Agrónomo, Universidad de los Llanos. OM.Sc. en Ciencias Ambientales.

5. Biólogo, Universidad Nacional de Colombia. Ph.D en Energía y Tecnologías del Medio Ambiente para el Desarrollo. Docente investigador.

\footnotetext{
* Transcripción textual
} 